\title{
Automatic Meter Reading System using CAN bus and Zigbee
}

\author{
Erapani Hanna ${ }^{1}$, G.Mani kumar ${ }^{2}$ \\ ${ }^{1}$ PG Student (M. Tech), Dept. of ECE, Chirala Engineering College, Chirala, A.P, India. \\ ${ }^{2}$ Assistant Professor, Dept. of ECE, Chirala Engineering College, Chirala, A.P, India.
}

\begin{abstract}
With the rapid development of automation and measuring techniques, automatic recording of the data in the meter reading instrument has gradually become the target of people whose working, living, and home conditions are of increasingly high level of intelligence. Meanwhile, utilities also hope that the development of new technologies to solve the problems they encountered in the practical work about cumbersome meter reading and no reliable protection of accuracy and real time; and enable both user friendly and improving public sector efficiency and management level. Existing wire-line meter reading system has a large number of risks. Wires are more complex, detrimental to adjustment and maintenance of the system. The long-term indoor and outdoor installation easily leads to aging, resulting in a risk of short circuit and breakage. For these reasons, it has become the industry very unresolved problem to design a remote meter reading system, with long-term reliance and convenient installation \& maintenance, which not only read data automatically but also monitor operation status. In this Paper we propose an automatic meter reading system which uses CAN Bus and Zigbee Technology to deliver the power consumption details to the sub station.
\end{abstract}

Keywords: Zigbee, Meter Reading, CAN

\section{Introduction}

With the development of wireless communication technology, in recent years there comes requirement for low cost equipment of wireless networking technology, called ZigBee. It is a short range, low-complexity, low cost, low power consumption, low data rate two-way wireless communication technology with high network capacity, short time delay, safety and reliance. Its main application areas include industrial controls, consumer electronics, car automation, agricultural automation, and medical equipment control. The core of this technology is established by IEEE 802.15.4 Working Group, and the ZigBee Alliance founded in 2002 is responsible for high-level applications, interoperability testing, and marketing [4].

The main methods of metering at home and abroad are: manual meter reading, IC Card prepaid meter, wire-line and wireless meter reading system. Manual meter reading has Been for decades, but with the implementation of one home one meter, drawbacks of this method of reading are more and more, like difficult entrance to home, low efficiency of fee settlement, etc. Along with the development electronic technology, IC card prepaid meter, which uses pay-before-use, favors to management sector. Since it does not need meter reading on site, partially solve the problem of manual meter reading because of no need to be on site. But some problems exist in the actual operation process: IC card meter is easily damaged due to its direct contact with user and no real-time monitoring. It also fails to avoid theft, damage, and fault of meter. Wire-line metering control system has the advantage of IC card, and at the same time can take full advantage of telephone network, power line network, a cable TV network, and RS-485 bus net, etc. It enables real-time metering, real-time monitoring, and real time control, and can also detect equipment damage, illegal use, etc. But there are some issues: piping, cable wiring, so it needs to design in advance. In addition, it also has problems of long construction period, high installation cost and maintenance cost, expansion of the system upgrade and compatibility with other network. ZigBee wireless meter reading refers to the use of short range wireless communication technology and computer network technologies to read and process metering data automatically. Wireless automatic meter reading technology can not only save human resources, but more importantly may improve the accuracy and real time of the meter, enabling management sector to access to data messages timely and accurately. No cable wiring can save human and material resources, so investment is considerably economical. Wireless communication links can be quickly built, engineering cycle significantly shortened, and has better scalability compared to a wire-line system $[2,3]$. If a fault occurs, only check wireless data module for causes quickly, and then restore the system back to normal operation [4].

\section{The Meter Reading System Model \& Design}

Main applications of ZigBee are within short range and data transfer rate among the various electronic equipments is not high. The typical transfer data types are periodical data (such as sensor data), intermittent data (such as lighting control), and repetitive low latency data (such as a mouse). 
Because Bluetooth, Wi-Fi, and ZigBee all belong to 802.15 protocols, technical characteristics have many similarities. Characteristics comparison is shown in Table 1. Since transmission distance of Bluetooth and Wi-Fi is less than $100 \mathrm{~m}$, it is a huge obstacle in the larger network. Thus it is difficult to form wireless communication network, while the maximum transmission range of ZigBee is $100 \mathrm{~m} \sim 1.5 \mathrm{~km}$, which is ideal for the establishment of the network. Secondly, it can only accommodate up to 8 nodes in a Bluetooth network, but a typical network needs more devices nodes, and ZigBee can accommodate 65536 nodes. Finally, module costs of Bluetooth and Wi-Fi are relatively high. In addition, the power consumption of Bluetooth and Wi-Fi compared to ZigBee is much higher, because ZigBee is low cost and very low power consumption. Although transmission data rate of Bluetooth and Wi-Fi is higher than that of ZigBee. But the 250kbit/s data rate of ZigBee is enough for use in automatic meter reading network, thus the ZigBee technology is selected.

Table 1 Technology Comparison

\begin{tabular}{|l|l|l|l|}
\hline \multicolumn{1}{|c|}{ Performance } & \multicolumn{1}{|c|}{ ZigBee } & \multicolumn{1}{c|}{ Bluetooth } & \multicolumn{1}{|c|}{ W-Fi } \\
\hline $\begin{array}{l}\text { Working } \\
\text { frequency }\end{array}$ & $\begin{array}{l}2.4 \mathrm{GHz} \\
868 / 915 \mathrm{MHz}\end{array}$ & $2.4 \mathrm{GHz}$ & $2.4 \mathrm{GHz}$ \\
\hline System resource & $4 \mathrm{Kbyte} \sim 32 \mathrm{Kbyte}$ & $250 \mathrm{Kbyte}$ & $1 \mathrm{Mbyte}$ \\
\hline Comm. range & $0.1 \sim 1.5 \mathrm{~km}$ & $0.1 \mathrm{~km}$ & $0.1 \mathrm{~km}$ \\
\hline Data rate & $250 \mathrm{Kbps}$ & $1 \mathrm{Mbps}$ & $11 \mathrm{Mbps}$ \\
\hline $\begin{array}{l}\text { Max. network } \\
\text { nodes }\end{array}$ & 65536 & 8 & 32 \\
\hline Wake-up time & $30 \mathrm{~ms}$ & $10 \mathrm{~s}$ & $3 \mathrm{~s}$ \\
\hline Encryption & 128 bits AES & 128 bits & SSID \\
\hline $\begin{array}{l}\text { Low power } \\
\text { consumption }\end{array}$ & Support & No support & No support \\
\hline
\end{tabular}

The wireless automatic meter reading system described in this article can send data from a number of digital watt meters to the collector through CAN bus communication protocol, and transfer the data to ZigBee Gateways through CAN bus network, then back to PC in RS-232 communication format via Zigbee as shown in Fig. 2. The wireless communication network in the middle uses JN5121 ZigBee modules produced by Jennic Company in combination with G-4500 GPRS remote communication devices developed by ICP DAS, forming a wireless communication network.

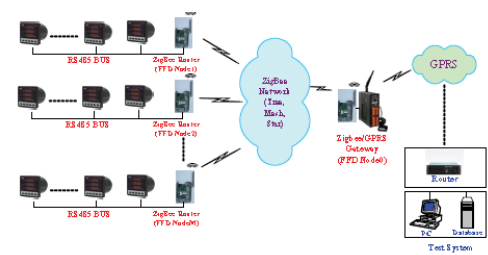

Figure 1 System structure of wireless automatic meter reading system

External terminal node module is mainly used for upgrade and improvement of existing electric meter network. The electric meter has RS-485 bus interface. Main tasks of the module are to access, read, and write the electric meter. Fig. 2 is the hardware block diagram of external terminal node. In order to communicate with meters, there is a chip for conversion of voltage level because the electric meter communication interface is RS485. This article will use MAX3485 chip, making it compatible with voltage level of JN5121.

ZigBee end device reads energy measurement information in multifunction electric meter by UART, and transmits them to the network coordinator via Zigbee wireless network, thus it can realize the wireless meter reading of the network coordinator. Meanwhile, the network coordinator can transfer clock-correcting command to multifunction electric meter through ZigBee end device to calibrate the system clock and power parameters. Therefore, the end device must be able to communicate with the multifunction electric meter in order to read data and the coordinator in order to send data. The coordinator also has to know which end node that sends the data so the data can be read accurately. Each of ZigBee modules has a unique 64-bit permanent address, so the addressing won't be much difficult. Fig. 3 shows the program flowchart of end device.

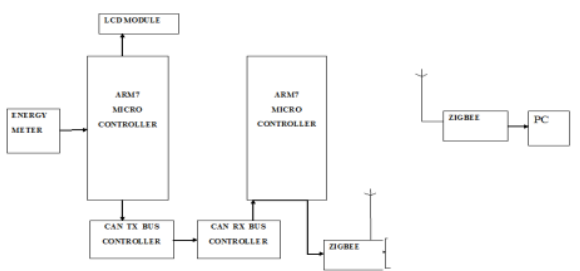

Figure 2 Hardware Block Diagram 


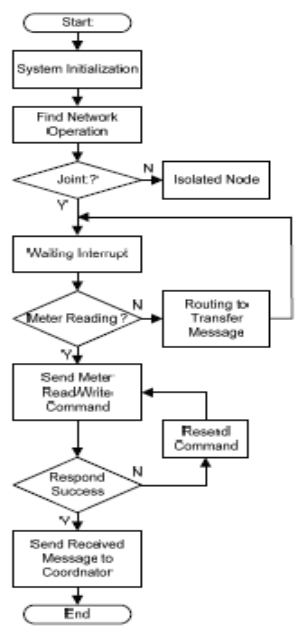

Figure 3 Program flowchart of the end device

The monitoring and management interface (that is $\mathrm{PC}$ ) of the wireless automatic meter reading system in this research is shown in Fig. 4. It is coded with the Visual Basic Program language developed by Microsoft. As a data receiver, it receives multifunction electric meter data including voltage, current, active power, power factor, and watt-hours. The communication port number on which the coordinator connects and the baud rate of the protocal can also be set on the interface. The automatic meter reading system considerably reduces both the difficulty of meter reading and human resources. Moreover, it also significantly improved the accuracy and instantaneity of data collection due to the use of digitalized management.

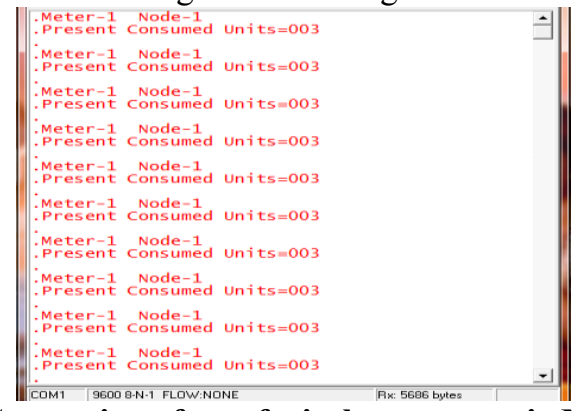

Figure 4 Monitoring System interface of wireless automatic Meter reading Unit

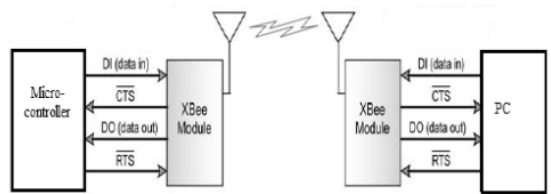

Figure 5 Zigbee Transmission Reception Pattern

The microcontroller data is transmitted through two separate boards i.e. ZigBee transmission module to the data collector or receiver module. The microcontroller used here is LPC2148 with $12 \mathrm{MHz}$ operating frequency. As shown in Fig. 2, the data receiving terminal of Zigbee XB_RX and data transmitting terminal $\mathrm{XB}$-TX are cross connected to the microcontroller corresponding transmitter and receiver terminals TxD and $\mathrm{RxD}$ respectively. No extra component like MAX 232 and MAX233 is required between these connections.

If the structure of UART (Universal Asynchronous Receiver Transmitter) system is completed, sending and receiving signal is possible using ZigBee, after installing necessary software. The RESET pin of Zigbee is used to provide an optional reset facility of user through a reset button. A transistor is used for this purpose.

The Zigbee Modules used in the interfacing boards, are engineered to meet IEEE 802.15.4 standards. It is low-cost, low-power, reliable 20 pin device that operates within the ISM $2.4 \mathrm{GHz}$ frequency band. It has 30 to 100 metre data transmission capability with rate of 250,000 bps. Zigbee modules operate in five modes. When not receiving or transmitting data, the RF module is in Idle Mode. The RF module shifts into the other modes of operation under various conditions.

In transmit mode serial data is received in the DI (data in) buffer and the data is stored in the DI Buffer until it can be processed. When the DI buffer is 17 bytes away from being full, by default, the module deasserts CTS (high) to signal to the host device to stop sending data (as shown in Fig. 6). CTS is re-asserted after the DI Buffer has 34 bytes of memory available. Smaller size data or low baud rate can be selected to avoid this state of overflow. In receive mode valid RF data is received through the antenna. When RF data is received, the 
data enters the DO (data out) buffer and is sent out the serial port to a host device. Once the DO buffer reaches capacity, any additional incoming RF data is lost. If RTS (hardware flow control) is enabled for flow control, data will not be sent out the DO buffer as long as RTS is de-asserted. Sleep Modes enable the RF module to enter states of low- power consumption when not in use i.e. not transmitting/receiving data for the amount of time predefined by the ST (Time before Sleep) parameter. To modify or read RF Module parameters, the module must first enter into Command Mode - a state in which incoming characters are interpreted as commands. The programming requires the installation of X-CTU software and a serial connection to a PC. The detail of the software is given in the next section. When communication occurs between two networked devices, each data packet contains a 'Source Address' and a 'Destination Address' field.

\section{CAN Architecture:}

CAN was first developed by Robert Bosch $\mathrm{GmbH}$, in 1986 when they were requested to develop a communication system between three ECUs (electronic control units) in vehicles by Mercedes. They found that an UART is no longer suitable in this situation because it is used in point-to-point communication. The need for a multi-master communication system became imperative. The first CAN silicon was then fabricated in 1987 by Intel.

\section{CAN standards:}

The original specification is the Bosch specification. Version 2.0 of this specification is divided into two parts:

Standard CAN (Version 2.0A): Uses 11 bit identifiers.

Extended CAN (Version 2.0B): Uses 29 bit identifiers.

The two parts define different formats of the message frame, with the main difference being the identifier length. There are two ISO standards for the difference is in the physical layer, where ISO 11898 handles high speed applications up to $1 \mathrm{Mbit} / \mathrm{second}$. ISO 11519 has an upper limit of $125 \mathrm{kbit} / \mathrm{second}$.

Format of a CAN message:

In a CAN system, data is transmitted and received using Message Frames. Message Frames carry data from a transmitting node to one, or more, receiving nodes.

The CAN protocol supports two Message Frame formats. The two formats are:

- Standard CAN (Version 2.0A)

- Extended CAN (Version 2.0B)

Most 2.0A controllers transmit and receive only Standard format messages, although some (known as 2.0B passive) will receive extended format messages but then ignore them. 2.0B controllers can send and receive messages in both formats.

\section{CAN 2.0A Format:}

A Standard CAN (Version 2.0A) Message Frame consists of seven different bit fields: frame.

- A Start of Frame (SOF) field. This is a dominant (logic 0) bit that indicates the beginning of a message

- An Arbitration field, containing an 11 bit message identifier and the Remote Transmission Request (RTR) bit. A dominant (logic 0), RTR bit indicates that the message is a Data Frame. A recessive (logic 1) value indicates that the message is a Remote Transmission Request (otherwise known as Remote Frame.) A Remote Frame is a request by one node for data from some other node on the bus. Remote Frames do not contain a Data Field.

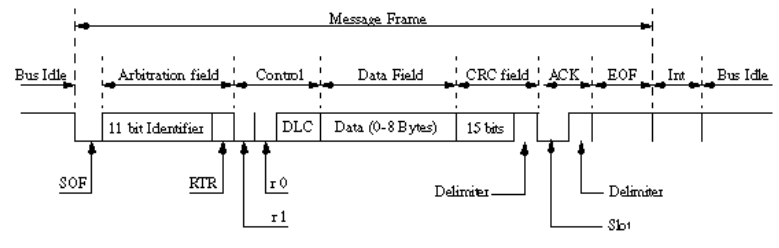

Figure 6 CAN Message Format

ZigBee wireless sensor nodes mainly consist the sensor unit, signal conditioning circuitry, microcontroller (MCU), RF modules MC13193, timers, memory and power management module and other components shown in Fig.2.

Micro-controller is responsible for collecting meter reading information and do some data conversion, responsible for controlling and managing the entire nodes; RF module MC13193 is responsible for 
the communication between nodes by a certain protocol ; the power module provides the necessary power for the nodes separately to run the various parts.

Sensor nodes are the basis unit of wireless sensor network; node stable running ensures the reliability of the whole network. Sensor node is comprised of data acquisition module, data processing module, wireless communication module, alarm module and the power module.

The data acquisition module is used for sensing, collecting information and converting to digital signals. According to the need for monitoring parameters of coal mine, the processor module is connected to gas sensor, pressure sensor, temperature sensor, and other kinds of sensor module, which is in charge of processing the data and coordinating the whole system.

The wireless communication module is mainly responsible for communicating with other nodes. In addition, the energy problem is the key problem, because once nodes exhaust the energy of the battery, which will drop out of the wireless sensor network, so power consumption of the wireless sensor network should be low as far as possible. Fig. 2 Shows the Block diagrams of the Present work.

\section{Control Logic:}

The control logic block controls the setup and operation of the MCP2515 by interfacing to the other blocks in order to pass information and control. Interrupt pins are provided to allow greater system flexibility. There is one multi-purpose interrupt pin (as well as specific interrupt pins) for each of the receive registers that can be used to indicate a valid message has been received and loaded into one of the receive buffers. Use of the specific interrupt pins is optional. The general purpose interrupts pin, as well as status registers (accessed via the SPI interface), can also be used to determine when a valid message has been received. Additionally, there are three pins available to initiate immediate transmission of a message that has been loaded into one of the three transmit registers. Use of these pins is optional, as initiating message transmissions can also be accomplished by utilizing control registers, accessed via the SPI interface.

\section{CAN Protocol Engine:}

The CAN protocol engine combines several functional blocks, shown in Fig 6 and described below.

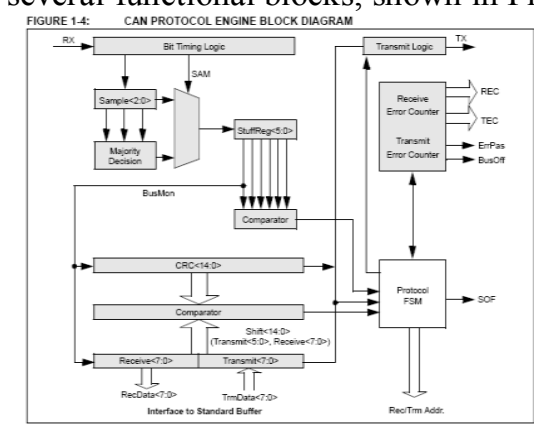

Figure 7 CAN Protocol Engine

\section{Conclusions}

In This paper, the implementation of a Meter reading monitoring monitor system is explained. The system is composed of PC monitors, meter reading nodes and base station nodes. This system based on wireless sensor networks, and hardware and software design of wireless sensor network are described in detail, this system can monitor the power consumption of various users at various parts of the building or various houses of a colony etc.,. Wireless sensor networks applied in monitoring of power consumption is a break through for the traditional methods and ideas, which improves the practical ability and flexibility of monitoring system.

The successful development of the wireless automatic meter reading system described in this article is based on the high performance, extremely low power consumption, high level of integration, and low price of ZigBee technology. The technology has strong market competitiveness. ZigBee wireless meter reading system uses short-range wireless communication and computer network technologies to read and process metering data automatically. Wireless automatic meter reading technology can not only save human resources, but also improve the accuracy and instantaneity of the meter reading. It enables management sector to timely and accurately access power consumption.

For the proposed wireless automatic meter reading system, wireless communication links can be quickly built, engineering period significantly shortened, and it has better scalability compared to a wired system. If a fault occurs, simply checking wireless data module can quickly find it out and restore the system in normal operation. 


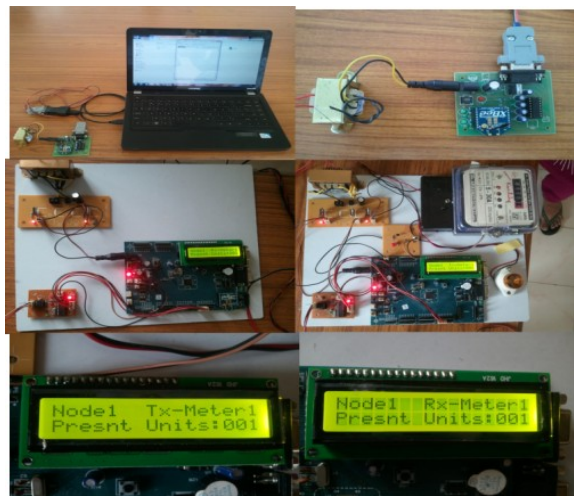

Figure 8 Designed Meter reading system prototype

\section{Acknowledgements}

The authors would like to thank the anonymous reviewers for their comments which were very helpful in improving the quality and presentation of this paper.

\section{References:}

[1] Satoshi Takahashi, Jeffrey Wong, Masakazu Miyamae, Tsutomu Terada, Haruo Noma, Tomoji Toriyama, Kiyoshi Kogure, Shojiro Nishio, "A ZigBee-based sensor node for tracking people's locations", proceedings of the 2nd ACM international conference on Context-awareness for selfmanaging systems, 2008, pp. 34-38.

[2] Sumi M, Ebrahim A. Soujeri, Rahim Rajan, Harikrishnan A. I, "Design of a zigbee-based RFID network for industry applications", proceedings of the 2nd international conference on Security of information and networks, 2009, pp. 111-116.

[3] Sharly Joana Halder, Tae Young Choi, Jin Hyung Park, Sung Hun Kang, Sin Woo Park, Joon Goo Park, "Enhanced ranging using adaptive filter of ZIGBEE RSSI and LQI measurement", Proceedings of the 10th International Conference on Information Integration and Web-based Applications \& Services, 2008, pp. 367-373.

[4] Hung-Cheng CHEN, Long-Yi CHANG "Design and Implementation of a ZigBee-Based Wireless Automatic Meter Reading System” PRZEGLĄD ELEKTROTECHNICZNY (Electrical Review), ISSN 0033-2097, NR 1b/2012, p. 63-68.

[5] Yu Hai-bin, Zeng Pengo Intelligent Wireless Sensor Network Systems [M). Beijing: Science Press, 2006.

[6] Zigbee Standards Organization, Zigbee Specification, Zigbee Document 053474r17, January 17, 2008

[7] S. Wei, L. Li-li, "Multi-parameter Monitoring System for Coal Mine based on Wireless Sensor Network Technology", Proc. international IEEE Conference on Industrial Mechatronics and Automation, pp 225-27, 2009.

[8] N. Chaamwe, W. Liu, H. Jiang, "Seismic Monitoring in Underground Mines: A case of Mufulira Mine in Zambia Using wireless Sensor Networks for Seismic Monitoring", Proc. IEEE international Conference on Electronics and Information Engineering, vol. 1(V1), pp 310-14, 2010.

[9] Qiao Ying-xu, Design of Wireless Sensor Networks Node Based OnTinyOS Operating System.The 3th International Conference on Computer Science and Education[C] 2008.7 1201-1204

\section{Authors Profile:}

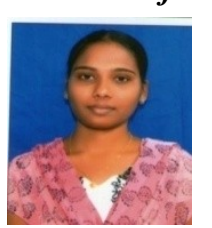

Erapani Hanna is Pursuing her M. Tech from Chirala Engineering College Chirala in the department of Electronics \& Communications Engineering (ECE) with specialization in VLSI \& Embedded systems.

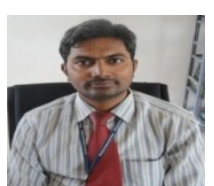

G.Mani kumar is working as an Assistant Professor in the department of Electronics \& Communication Engineering in Chirala Engineering College, Chirala. He has 5 years of teaching experience along with one year industrial experiance. 